\title{
LA TIERRA: \\ UN PLANETA DISEÑADO \\ PARA LA VIDA
}

Hovile Enrapar RLAS RareGO

Cuando tomamos como fuente de información a las ciencias geológicas para obtener una visión medular en el tema de estudio de nuestro planeta, notaremos que muchos puntos de vista con relación a creencias de la Fé, discrepan con claridad, sobre aspectos biológicos y filosóficos principalmente; sin embargo, al examinar cuidadosamente los mecanismos que actuan en la Tierra y que hacen de ella un planeta especial y muy dinámico en el contexto del sistema solar, simplemente confirmamos que las esferas interactuantes, -hidrósfera, atmóstera, biosfera y litosfera), se combinan y responden de manera continua $e$ indefinida en medio del tiempo, que implacable creará la sucesión de fenómenos que dan lugar a los cambios que se producen incesantes sobre la faz de la Tierra. Nuestra visión, nos permite apreciar localmente en cualquier linea de costa, cómo se producen estas interacciones cuando se encuentran las rocas, el agua, el aire y la presencia de organismos vivos como el hombre, ante lo cual se experimentan sensaciones de diversa naturaleza en cada persona.

De acuerdo a las dataciones radiométricas del sistema solar y la Tierra por extensión, se le asigna una edad de 4.500 millones de años. en cuyo contexto se ha fabricado una cronología geológica que desprende los siguientes postulados de la estratigrafía y la geología histónica:

- La edad de la Tierra se calcula en miles de millones de años.

- El aspecto físico de la Tierra ha sufrido muchos cambios, grandes y pequentos, durante el tiempo geológico.

- Los seres vivos poblaron la Tierra hace centenares de millones de años. Una vez que apareció la vida, ésta no ha dejado de existir.

- La evolución de la vida no ha sido uniforme; muchos organismos han estado cambiando constantemente, mientras que los más primitivos y simples han persistido en forma estacionaria.

- Ninguna especie, una vez extinguida, ha vuelto a aparecer.

Los fósiles, representan sólo una pequeña parte de la vida de cada periodo. 


\section{EL MAPA GEOLOGICO}

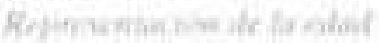

\section{ESCALA CRONOESTRATIGRAFICA}

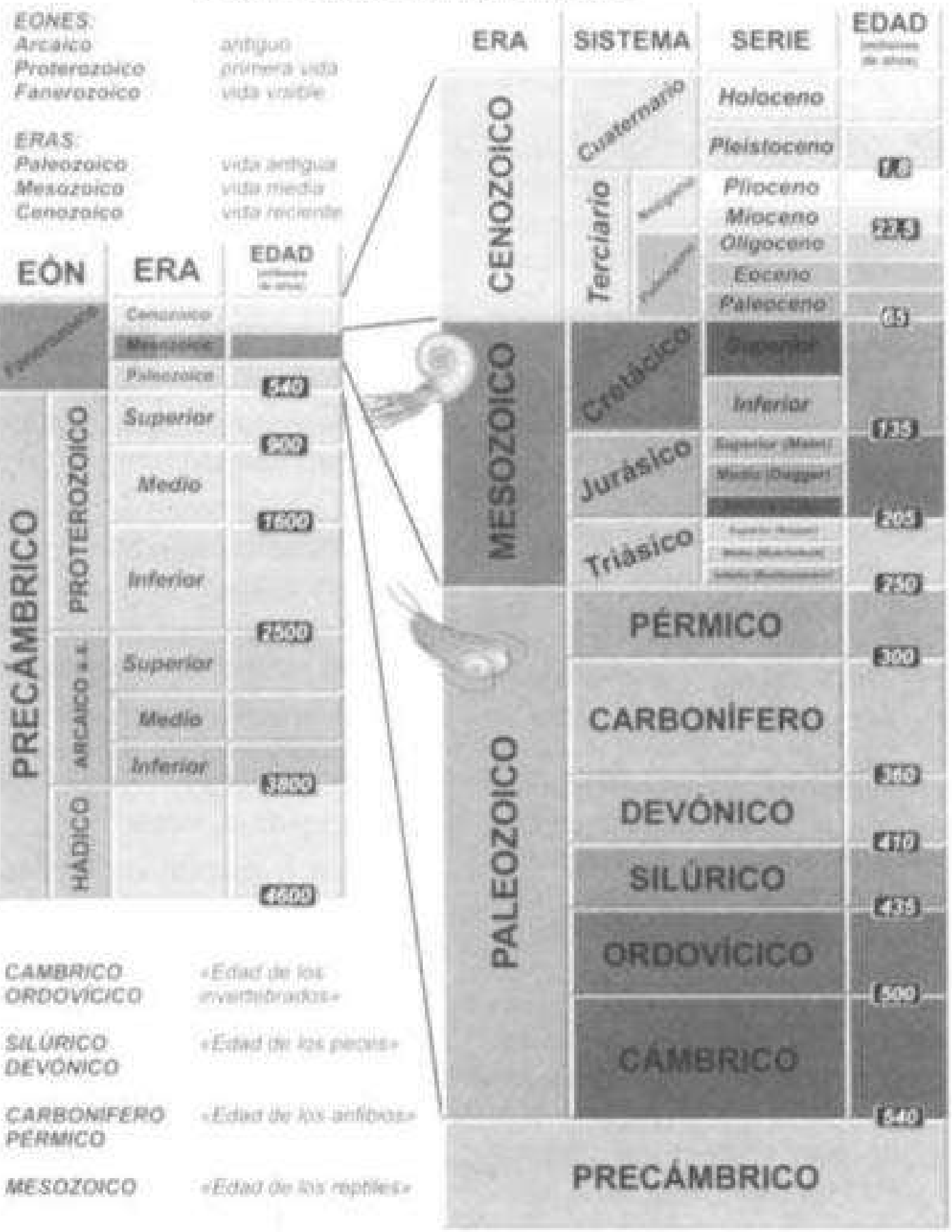

AGURA ins

Evala cronoestratigrafica en far aue se muesiran ies diferentes colores que sueler she utilizaden nara determinar la kdad de un material. Eitos colores bueden wavar en fincion del pais o de la verie cartografura.

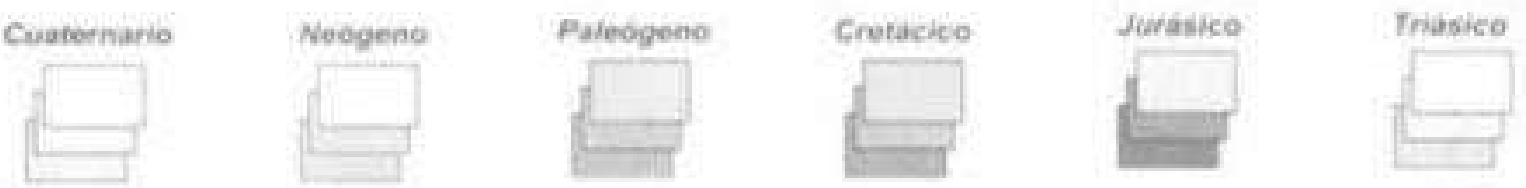

yeuma in.6

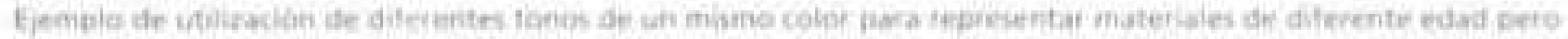
pertenerientes a un mismo witema o a una mivma werie. 


\section{EL ORIGEN DE LA TIERRA, PLANETA AZUL}

La Tierra es el tercer planeta del sistema solar, al que se le contempla azul desde el vasto espacio, cual inmensa burbuja esférica que "...flota sobre la nada", tal como lo expresara un hombre fiel a Dios: Job, cap. 26, vers. 7 de la Biblia, unos 3.000 años antes que el hombre confirmara la esfericidad del planeta.

Muestra esta tonalidad, porque es el único planeta que posee una envoltura gaseosa liamada atmósfera y una sustancia liquida milagrosa, sustento de toda vida denominada hidrosiera, la misma que se halla también en estado solido y gaseoso, formando parte de nuestros oceanos, lagos y rios; asi como de imponentes glaciares en los polos y zonas altas del globo. El vapor de agua en su forma gaseosa, se concentra en las nubes mostrando la singularidad de ser el único planeta que muestra el brote de la vida en medio de complicados, pero bellos a la vez, geosistemas interrelacionados.

La naturaleza ordenada de nuestro sisterna solar, conduce a la mayor parte de astrónomos, a concluir que todos sus componentes se formaron esencialmente al mismo tiempo y de la misma materia primordial que el Sol. Este material formó al inicio del sistema solar, una gran nube de polvo y gases denominada, nebulosa primordial o primitiva, dando paso a esta hipotesis moderna que sustenta los procesos que son bastante aceptados por los geólogos actuales.

\section{LA NEBULOSA PRIMITIVA}

- Se sostiene que todos los cuerpos de nuestro sistema solar se formaron a partir de una enorme masa difusa, en forma de nebulosa compuesta elementalmente por $\mathrm{H}$, He y una pequeña parte de elementos más pesados como $\mathrm{Fe}, \mathrm{Mg}$. y Ni.

Alrededor de 5.000 millones de años atrás, esta inmensa masa combinada de elementos ligeros con particulas pesadas, empezo a contraerse bajo su propio influjo gravitatorio, al mismo tiempo que en forma gradual, giraba muy lentamente.

\section{HIPÓTESIS DE LA NEBULOSA PRIMITIVA}
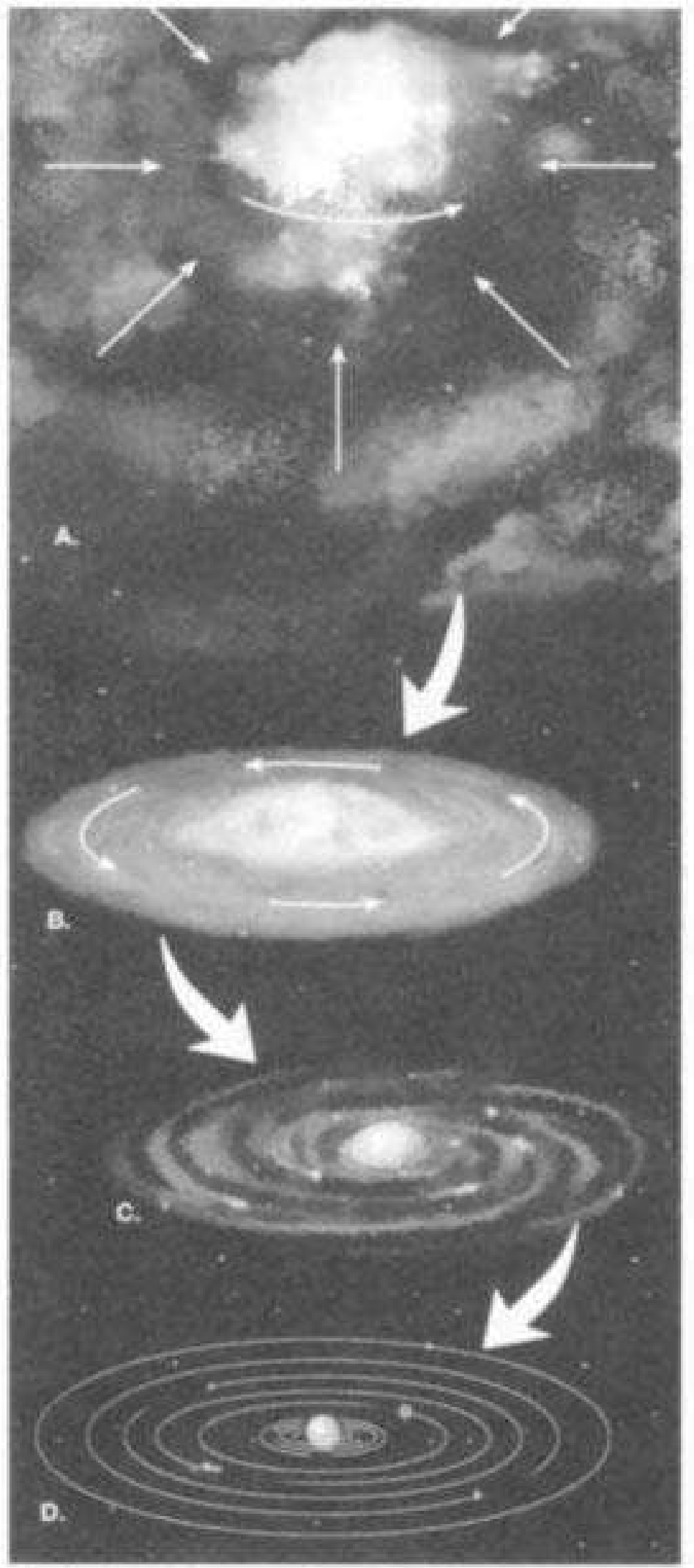

A. Una enorme nube de polvo y gases en rotación, nebulosa, empieza a contraerse.

B. La mayor parte del material, es gravitatoriamente impulsado hacia el centro. originando el Sol. Sin embargo, debido al movimiento rotacional, algo de polvo y 
gases permanece en orbita alrededor del cuerpo central en forma de un disco aplanado.

C. Los planetas empiezan a formarse a partir del material que está en órbita dentro del disco plano.

D. Con el tiempo, la mayor parte de los restos se fueron reuniendo en los nueve planetas y sus lunas o bien, expulsados al espacio por el viento solar.

- A medida que se acentuaba dicha contracción, transmitia energia de rotación más acelerada, de manera tal que la nebulosa tue adquiriendo una forma de disco y los elementos inmersos, gravitatoriamente son impulsados hacia el centro, donde se van concentrando altas temperaturas dando origen al futuro Sol.

- Los cuerpos menores que se forman por la colisión de particulas, dan lugar al conjunto de planetas y demás astros que componen nuestro sistema solar.

- Conforme estos planetas acumulaban más material, el espacio formado entre ellos empezaba a tornarse cada vez más claro, de tal modo que la luz del material que formaba el sol, brillaba intensamente llegando a la superticie de los planetas ya formados y no habia obstáculos para calentarlos.

- Las elevadas temperaturas en los planetas más internos. Mercurio. Venus, Tierra y Marte, los hacen incapaces de conservar cantidades apreciables de materiales ligeros como hidrógeno, helio, amoniaco, melano y vapor de aqua, que se evaporaron de sus superficies, siendo barridos por el viento solar.

- A distancias mayores de la órbita de Marte, las temperaturas son mas frias: consiguientemente los grandes planetas exteriores, Júpiter, Saturno. Urano y Neptuno, acumulan grandes cantidades de elementos ligeros como el $H$, procedentes de la nube primitiva. Se cree que la acumulación de esas sustancias gaseosas, generaron los tamaños comparativamente grandes y de baja densidad en las masas de los planetas exteriores
Con relación a los movimientos independientes de cada planeta-rotación y traslación- muly particular en cada uno, no se conoce con precisión sus objetivos; salvo en nuestro planeta, sabemos que es la dinámica viva que responde a todos los procesos que experimenta, desde las poderosas fuerzas que en forma continua modifican su superficie, hasta la interacción de sus geosistemas que en conjunto. integran la suma de enigmas que dan paso a las condiciones de aparición de los gérmenes del portento de la vida, en toda su magnitud

El último planeta atrapado en la familia solar, ahora dectarado como no planeta, se halla en el extremo orbital eliptico más alejado del Sol; es pequeño y misterioso, mostrando en su superficie, una gruesa capa de agua congelada.

\section{LA VIDA... ¿CÓMO SE INICIÓ?}

Un punto crucial en la vida de cada hombre o mujer, ha sido y seguirá siendo esa constante enigmática ¿cuál es el origen de la vida? Desde un punto materiai, A. 1. Oparin, cerrado defensor de fa teoria evolucionista, en su obra El origen de la vida $(1978,140)$ da por sentado que ésta se inició por efectos de la movilización conspicua del carbono que:

"Se hallaba disperso en atomos libres por la atmósfera, luego se hallaria formando parte de los hidrocarburos originados en la superficie de la Tierra. Posteriormente, estos hidrocarburos dieron derivados oxigenados y nitrogenados que dieron lugar a las sustancias orgànicas más sencillas: luego en las aguas de los primitivos océanos, esas sustancias crearon o formaron cuerpos más complicados surgiendo las proteinas y sustancias similares que vienen a constituir la materia de la cual estän formados los animales y ios tejidos vegetales"

También sostiene que en un principio, los senes vivos no tenian estructura celutar, to que posteriormente en una determinada etapa del desarrollo de la vida, recién surge la unidad viviente llamada célula formando inicialmente organismos unicelulares y posteriormente organismos pluricelulares que poblaron la Tierra. Concluye que las ciencias naturales modemas con sus grandes 
adelantos han aicanzado descubrir las leyes que precedieron el ongen y el desarrollo de la vida. Sin embargo, notaremos que al mencionar de leyes que rigen el origen de la vida, implicitamente sostiene una sucesión organizada de acontecimientos que hicieron posible la aparición de la vida en la Tierra, luego nos preguntamos. ¿quien fue el autor de dichas leyes?

Si examinamos teorias más recientes. observaremos divergencias en sus hipótesis, mientras algunas sostienen la generación espontánea de la vida, otras postulan que la vida se originó en el espacio cósmico y fue descendiendo hacia la Tierra. En tal sentido. notamos que éstas y otras teorias van a confundir at comùn de las personas. Si se recurre al registro fósil, se vera que no existe un desarrollo gradual de un tipo de vida a otro, de acuerdo a lo que sostiene la teoría evolucionista. porque de tener sustentos confiables, se hallarian estas pruebas diseminadas en todo lugar de la Tierra; además, si analizamos con atención las ideas de Ch. Darwin, tanto tiempo aceptadas de la supervivencia del más apto o la llamada: selección natural, donde la naturaleza más vigorosa del individuo permitiria su supervivencia, pero en más de 140 años las evidencias muestran que aunque los más aptos sobrevivan, ello no explica cómo se originaron: un perro puede ser más apto que otro perro. pero esto no explica cómo llegó a ser perro y toda su descendencia seguirá siendo de perros y no de otro animal, lo que significa también que existirán variedades de la especie, que no es igual a especies diferentes.

A) tratar el tema con una dosis de sutileza. percibimos que la vida se halla alrededor de todos nosotros; en el zumbido de los insectos. los pájaros y sus melódicos trinos, el crujido de las maleza causado por los animalillos que huyen asustados, en las heladas reglones polares y en áridos desiertos secos; se halla en el mar, desde sus partes más superficiales, hasta sus más recónditas profundidades; en la atmósfera flotan minusculas criaturas y bajo nuestros pies. se mueven millones de microorganismos en afán de fertilizar lo suelos y dar paso al crecimiento de plantas verdes que son el sustento de otras variadas formas de vida. En resumen, la Tierra esta colmada de vida tan abundante que no llegamos a formar un cuadro entero de todo lo existente. Nos preguntamos entonces ¿cómo es esto? La incipiente y no muy definioda aparición de organismos unicelulares y pluricelulares posteriores, pudieron dar lugar a la formación de cientos de miles, de especies tan variadas que pueblan nuestro planeta?

En forma más particular, ¿cuál fue el principio de la humanidad? ¿Venimos como descendientes de bestias simiescas o somos producto de una portentosa creación? Preguntas similares se han oido por mucho tiempo y las respuestas flotan en la mente de muchas personas, Mas estas mismas personas se hallan en capacidad de discernir dichas interrogantes, al contemplar el orden de la naturaleza en toda su extensión. El punto de vista que podamos concluir en este polémico entomo, intluye sin duda en nuestras actitudes hacia la vida y el futuro, asi como puede afectar el curso de la historia y nuestro lugar en ella:

\section{A TIERRA: UN PLANETA DISEÑADO PARA LA VIDA}

Nosotros planteamos esta temática por la complejidad con que se presentan los geosistemas relacionados a nuestro planeta; tas ciencias geológicas ni otras cercanas son incapaces de satisfacer la interrogantes que brotan de sus profundidades. En esta sección, intentamos resumir algunos aspectos que le dan a la Tierra una particularidad y un privilegio único, de ser fuente de la milagrosa vida.

\section{La estructura del globo terrestre}

Al despejarse completamente las ultimas particulas de la nube primitiva, cada planeta habria adquirido una estructura definida. Nuestro Planeta, presenta tres capas concéntricas bastante notables: corteza, manto y núcleo.

Corteza. Es la parte superficial mejor conocida por el hombre, se halla constituida por tres tipos de rocas: igneas, sedimentarias y metamóricas. En su contexto, presenta gran rigidez y estructura complicada, Bajo los océanos su espesor varia entre 5 a $10 \mathrm{~km}$. y debajo de los continentes hasta más de $50 \mathrm{~km}$. Sus elementos constitutivos importantes son: O, Si, Al, $\mathrm{Fe}, \mathrm{Ca}, \mathrm{Na}, \mathrm{K}$, y Mg.

En la corteza, se pueden distinguir dos capas: una superior con predominancia de granito, y en la inferior con predominancia de basalto. Esta parte de la Tierra es el lugar donde se registran los más variados procesos 
geodinamicos producto de la interacción de sus geosistemas: atmósfera, hidróstera, litóstera y biostera. La zona de contacto entre la corteza y el manto recibe el nombre de Discontinuidad de Mohorovicic.

Manto. Se extiende desde las bases de la corteza, hasta los $2900 \mathrm{~km}$ de profundidad, se halla interrumpida por una franja Ilamada: capa de velocidad reducida, se ubica entre 50 a 200 $\mathrm{km}$. En esta franja, tas ondas sismicas se propagan a velocidad mucho menor que ef resto del manto; las partes inferiores son cada vez más densas y sus temperaturas y presiones son aitisimas. El $82 \%$ del volumen terrestre está contenido dentro del manto y se halla constituido por silicatos de magnesio, hierro. calcio y aluminio principaimente. La zona de contacto entre el manto y ef núcleo, se conoce como Discontinuidad de Gutenberg.

Núcleo. Se halla formado por los elementos más pesados de la nube primordial (hierroniquel). los que debido a su densidad y gravedad, ocuparon la parte centrica en dos niveles: nucleo exterior, que llega hasta los $5.100 \mathrm{~km}$, con temperaturas de $4.000^{\circ} \mathrm{C}$, y el nùcleo interno que ocupa el centro de la Tierra a $6.370 \mathrm{~km}$ y de $4.500^{\circ}$ a $6.000^{\circ} \mathrm{C}$, respecfivamente. Los investigadores, sostienen que el núcleo externo es la unica capa fluida que compone la Tierra, el cual por efectos de la rotación que experimenta nuestro planeta, amana su polaridad conocida.

Cuando los astrónomos y otros investigadores, concluyen que todos los demals planetas del sistema solar se encuentran deshabitados y que sólo ta Tierra posee las singulares cualidades de haber sido construida con sistemas bastante complejos que suministran fuz, aire, calor, agua y alimento en delicado equilibrio para sustentar a todos los organismos que la habitan junto con sus procesos de reproducción en orden a la preservación de su especie, observamos entonces que cuanto más es examinado el planeta y la vida que lo puebla, obtenemos como repuesta que el diseño no sólo es excelente. sino perfecto: nótese los siguientes delalles.

Con relación a la distancia al Sol. Entre las muchas condiciones que son vitales para la vida en la Tierra, se tiene que la fracción de energia que nuestro planeta recibe, es la precisa para el sustento de todas las especies. Es decir que a la distancia media de $149.600 .000 \mathrm{~km}$, se dan esas condiciones. Si la Tierra estuviera ubicada mucho más lejos o mas cerca, las temperaturas serian extremas para el sostenimiento de ta vida. Por otro lado, la velocidad orbital que experimenta la Tierra, aproximadamente $107.200 \mathrm{Km} / \mathrm{h}$, es la adecuada para contrarrestar la atracción gravitatoria del Sol y mantenerse a una distancia debida. Nuestro planeta, tambien gira sobre su eje cada 24 horas manteniendo una inclinación de $23^{\circ} 27$. lo que permite en forma periódica, establecer los cambios estacionales, haciendo que la Tierra se muestre con una cubierta diferente en primavera, verano, otono e inviemo, cada una de las cuales destacando por sus bellas particularidades.

Nuestra Atmósfera. Unica en el sistema solar, contiene porciones de gases combinados que son vitales para la vida; los gases componentes, por si solos, algunos son mortales. El O compone el $21 \%$ del aire respirable; si la atmóstera fuera $100 \%$ de O. se tornaria tóxico y contribuiria a que sean más inflamables los materiales combustibles, El $\mathrm{N}$ compone el $78 \%$ de la atmóstera, y en el curso de tormentas y descargas eléctricas, los rayos que se producen, permiten combinarse parte del $\mathrm{N}$ con el $\mathrm{O}$. La liuvia transporta al terreno estos compuestos, donde las plantas los utilizan como tertilizantes.

El $\mathrm{CO}_{2}$ (dioxido de carbono) compone menos del $1 \%$ de la atmóstera y esta pequeña cantidad, permite que las plantas los absorban del aire para liberar $\mathrm{O}$; si el $\mathrm{CO}$ fuese abundante en la atmóstera, seria mortal para las personas y animales; si fuese mucho menos del que existe, no sustentaria la vida vegetal. Entonces observamos que la atmósfera no sólo sostiene la vida, sino que sirve como una envoltura o capa protectora de las dañinas radiaciones solares y de los cuerpos que liegan a impacta: en la Tierra en forma de meteoritos, aparte que nos impresiona porque en ella se producen espectaculos originados por fenómenos como los celajes y otros que producen vivaces destellos,

El agua. sustancia milagrosa. Nuestro planeta, muestra el privilegio de poseer en abundancia, la maravillosa sustancia liquida llamada agua en sus tres estados físicos: gaseoso, en forma de vapor de agua; liquido. como grandes masas que forman los oceanos 
lagos y rios en forma de corrientes de agua. También como sólido, hielo, que ocupan en grandes extensiones en las zonas polares y algunas latitudes altas de la Tierra, formando imponentes glaciares. Todo ello de acuerdo a la variedad de temperaturas existentes en el globo: reconocemos que el agua, es el sustento principal de toda forma de vida, donde hay agua. hay vida, reza una frase popular: la acción del hombre en el movimiento de las máquinas para la industria, produce incesante consumo de combustible, lo que a su vez genera en la atmósfera el denominado efecto invernadero que junto tal vez a una época secular, haya originado una elevación de la temperatura global, propiciando el retroceso de nevados y glaciares existentes en muchas partes del globo, poniendo en grave peligro el equilibrio en las formas de vida, lo que debe llamamos a profunda reflexión, para valorar toda fuente de este preciado liquido.

La Tierra Productiva. Es impresionante conocer a quièn diseñó también el suelo del planeta Tierra, con toda su potencialidad para el crecimiento y desarrollo de la incontable variedad de plantas, donde la luz del Sol, el dióxido de carbono del aire, el agua y las sustancias minerales del suelo se combinan sabiamente para producir el alimento, convirtiendo los elementos químicos del suelo en sustancias asimilables por los organismos tanto humanos como ef de los animales. ¿Podemos imaginar los multiples productos alimenticios de origen vegetal existentes en la faz de la Tierra, desde los tubérculos, hasta las sabrosas frutas... alimentos que también evolucionaron paralelamente al hombre...?

\section{Colofón}

- El intelecto de cada persona es la facultad de la razón que nos hace percibir que nos hallamos frente a algo, a veces inexplicable. si pudiera tener la certeza de un tema tan cientifico o idealista, pero basado en aspectos sencillos y lógicos, contaria con los argumentos para defender mi posición.

a En múltiples ocasiones, el tema ha entrado en polémica, concluyendose en dos posiciones extremas: las personas que centran el tema desde una lógica de sencillez y Fé profunda, que se orienta a la existencia de un Dios creador, y los escépticos que al no ver esa logica. fundamentan la imposibilidad de la existencia de Dios.

a Frente a las disyuntivas que se presentan, es necesario brindar los respetos frente a quienes los sostienen, porque tan cientifico es lo material, como también lo es, la parte de quienes comparten sus ideas.

\section{BIBLIOGRAFIA:}

DAFWIN, Charles

1958 "The Origin of Species".

KENNETH F., Weaver

1974 "The incredible Universe".

OPARIN A.I.

1978 "El Origen de la Vida".

PIVERA MAURO

1976 "Compendio de Geología Física".

SAGAN, Carl

1980 "Cosmos".

TARBUCK Y LUTGENS

1999 Una Introducción a la Geologia Fisica", 


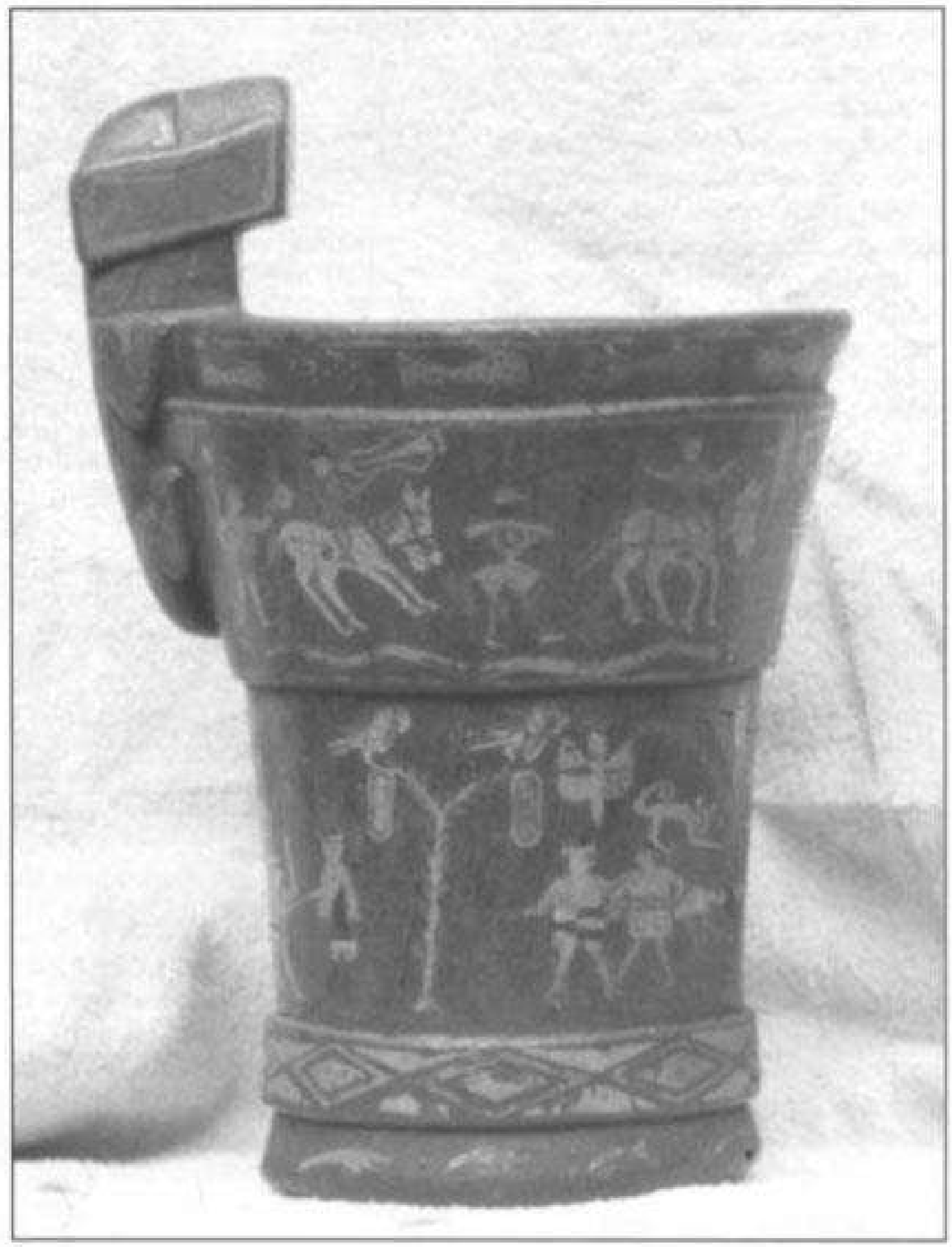

Vaso de Madera utilizado para libaciones con chicha, de uso cotidiano y Ceremonial Siglo XVII - Museo inka de la UNSAAC. 